\title{
Musical Auditory Perception in Piano Performance and Teaching
}

\author{
Song Yu \\ Xi`an conservatory of music, Shanxi Xi’an, 710061
}

Keywords: piano performance; teaching; musical auditory perception

\begin{abstract}
In the teaching of piano in China, teachers pay more attention to the mastery of students' skills. For the auditory perception in music creation and performance, there is no targeted practice, which lead students to master learning skills and performance skills through piano performance, and the ability to evaluate and appreciate music. Based on the current piano teaching in China, the lack of emotions and appreciation reduces the students' enthusiasm for piano learning and the improvement of artistic accomplishment. This paper analyzes the importance of music auditory perception to piano teaching in China's piano performance and teaching and also points out the importance of music auditory perception to piano teaching. It points out the importance of students' comprehensive quality and imagination cultivation in piano teaching, how to improve piano performance and teaching music. The training of auditory perception enhances the mastery of piano performance skills and the development of piano teaching.
\end{abstract}

\section{Introduction}

Piano teaching in music creation is the focus of music majors in colleges and universities in China. Teachers pay more attention to practice in the teaching process. Theoretical knowledge and skill training do not pay much attention to the perception of music and auditory. In other words, they focus on the mastery of students' skills, but emotions and appreciation are obviously insufficient are less. Although the students' music performance is good, the lack of music creation and improvement, technology and art have not been effectively integrated, making students' piano performance skills stay in form, it is difficult to achieve performance and learning effects. Musical auditory perception can effectively improve students' comprehensive quality and imagination, so that students can experience the emotions in the works through music rhythm and melody, promote the substantive performance of music performance and performance, and cultivate piano talents with creative and innovative consciousness.

\section{The importance of musical auditory perception in piano performance and teaching applications}

\subsection{Classification and characteristics of musical auditory perception}

Musical auditory perception mainly consists of two major categories. One is internal auditory perception, which mainly refers to the state of music psychology. Through the gradual improvement of the cognitive ability of the performer, the mastery of the playing knowledge and the mastery of culture and skill can enhance the player's music. The sensitivity of the sound, that is, the so-called beat and rhythm, feel the emotion and connotation of the music. Mainly through the construction of musical intentions, strengthen the understanding and thinking of music, the importance of internal auditory perception lies in re-creation, just like Beethoven, even in the case of deafness, can create the works of "Fate" and "Hero" To realize the creation of piano music, it can be said that internal auditory perception is the embodiment of the musician's musical feeling. Second, external music hearing is a physiological phenomenon. As long as people with normal hearing have the ability to feel sound, many piano players can judge the correctness of the performance process through external auditory perception. In the process of piano performance and teaching, teachers do not pay much attention to the teaching of internal auditory perception. The external auditory perception is mainly combined with skills, so that students can judge the accuracy of performance through 
hearing, but this can only reproduce piano music, not Being able to create piano music and realize the combination of internal hearing and external auditory perception can build a perfect musical intention and music connotation judgment, and realize the re-creation of music works. Musical auditory perception is characterized by orientation and memory, and the performance is judged by external auditory perception ${ }^{[1]}$.

\subsection{The importance of piano performance and teaching applications}

Musical auditory perception plays an important role in piano performance and piano teaching. It can effectively stimulate the musician's musical nerves and start from the foundation of piano performance to train students' basic piano performance. The first is to effectively use music to understand the music, to sense the music beats and melody with external hearing, to grasp the overall direction of the work and the connotation of the content, to strengthen the aesthetic and appreciation of music, and the continuous intensive training can even improve the harmony and the understanding of the instrument, the experience of playing and the progress of playing skills. Secondly, through the internal auditory music, the musician's music knowledge, music skills and aesthetic development can be improved, and the musical auditory perception ability can be enhanced. It can effectively stimulate the performer's creative inspiration to promote the control and perception of music, and form the creative ability and innovative consciousness of the musical works. To achieve the desired performance. The third is to use the musical auditory to realize the guidance of piano performance and practice. The basis of piano performance and teaching is practice, strengthen the musical auditory perception ability, improve the understanding of their own playing ability, self-find and solve problems, improve their playing ability and composing. Appreciation, etc., through the perceptual role of music throughout the teaching process, can improve the beginners' grasp of the rules of the vocalization of the keys, improve the emotional and artistic conception of the piano playing skills, and promote the understanding of musical hearing and the works ${ }^{[2]}$.

\section{The application of musical auditory perception in piano performance and teaching}

\section{1 exercise accompaniment ability, strengthen music imagination}

At present, in the piano teaching in China, the cultivation of students' auditory perception is not taken seriously, and their accompaniment ability is obviously insufficient. As a result, students are in an independent state in performance, and it is difficult to cooperate with the integrated music to play the works. Strengthen the training of musical auditory perception, effectively exercise the accompaniment ability, letting the students perform with other performers through group piano teaching, improve the students' auditory perception training and grasp the overall performance through systematic and practical piano accompaniment. From the point of view of accompaniment practice teaching, the rhythm is correctly grasped to perceive the performance process of the companion, and the students' musical auditory perception is invisibly exercised, and the accompaniment ability and the perceptual effect of music are mutually enhanced. Piano teaching focuses on cultivating students' musical imagination ability, making full use of music auditory perception to strengthen students' musical imagination. As far as the current music imagination ability of piano teaching in China is concerned, its innovation consciousness and creative ability are insufficiently cultivated, through the background and knowledge of the work. Explaining to improve students' understanding of the works does not allow students to experience the emotions and connotations of the works from the perspective of substantive performance and appreciation. Therefore, it is impossible to enhance students' understanding of auditory memory and musical emotions. Taking the silent practice as the core, let the students hear the silent performance connection on the keys and strengthen the emotional re-creation of the music. Combining only the connotation to analyze the works, the teacher needs to formulate a scientific and systematic exercise plan, in order to shape the artistic conception of the music works. And let the students stay on top of the artistic conception and realize music re-creation. Therefore, in the teaching process, it is 
necessary to pay attention to the students' musical listening and perceptual ability and the emotional experience of external music works. Through the accumulation of music and the enhancement of internal auditory services, the artistic conception and innovation consciousness are gradually improved $^{[3-4]}$.

\subsection{Improve overall quality and strengthen playing skills}

The students' understanding and innovation of music works is the foundation of the improvement of students' performance skills. The purpose of improving the overall quality is to strengthen artistic accomplishment. The mastery of playing skills in piano teaching is the focus of teacher teaching, but it is mainly through the teacher playing students to imitate the playing skills, which does not reflect the subjective initiative and enthusiasm of students, so it is difficult to achieve flexible application of skills, all in a fixed performance. Using fixed skills, it is difficult to achieve innovation in the performance process, playing a composition that is in line with his own emotional state, so the emotional expression ability and the form of performance of the work are seriously inadequate. Strengthen the training of students' music perception ability, improve students' response to piano performances, and use the same piano performances to allow students to express their emotions and reproduce their performances, which can fully enhance the performance of music performance and emotional expression, and promote students' In the performance, different emotions, such as depression, tension, joy, jealousy, etc., are used to adjust the students' understanding of the culture, meaning and meaning of the music works, and then introduce the historical background of the works to students. Feel the emotions of the music again. The accumulation of musical auditory sensibility lies in the understanding of musical works. Through the different types and the same types of works in the teaching process, students can improve their understanding of the music works and the overall quality of the students through memory deepening, and adjust the students' life. Viewing values and feeling the potential value and connotation of music, and improving students' grasp of the overall trend of composition ${ }^{[5]}$.

\section{Conclusion}

In general, various types of piano music are used in the teaching process to train students' judgments on the performance of the music, to improve the sensitivity of the students to the rhythm of the music, and to correct the mistakes in the music performance. In the process of playing music, it strengthens the memory of music melody and rhythm, gradually expands the player's understanding and understanding of the music rhythm, and grasps the overall trend of the music to show the characteristics of music listening and orientation. Therefore, the training of musical auditory perception needs to improve the musical auditory perception through the long-term systematic teaching according to the content of the music and the expansion of the melody.

\section{References}

[1] Fu Ronghua. Analysis of the Innovative Piano Performance under the Musical Hearing Perception [J]. Journal of Heihe University, 2018, 9(06): 169-170.

[2] Li Yitong. The Important Role of Musical Auditory Perception in Piano Performance and Teaching [J]. Art Research, 2018(02): 176-177.

[3] He Yang. The important role of musical auditory perception in piano performance and teaching [J]. Popular Literature, 2016 (23): 191.

[4] Zhao Yang. The important role of musical auditory perception in piano performance and teaching [J]. Voice of the Yellow River, 2017 (05): 45.

[5] Li Wei. The Role of Musical Auditory Perception in Piano Performance and Teaching [J]. Contemporary Music, 2017(11): 67-69. 